hep-th/9702203

RU-97-09

UCB-PTH-97/07

LBNL-40003

\title{
Issues in $\mathrm{M}($ atrix) Theory Compactification
}

\author{
Michael R. Douglas ${ }^{1}$, Hirosi Ooguri ${ }^{2,3}$ and Stephen H. Shenker ${ }^{1}$ \\ ${ }^{1}$ Department of Physics and Astronomy \\ Rutgers University \\ Piscataway, NJ 08855-0849 \\ mrd, shenker @physics.rutgers.edu \\ ${ }^{2}$ Department of Physics \\ University of California at Berkeley \\ Berkeley, CA 94720-7300 \\ ${ }^{3}$ Physics Division, Mail Stop 50A-5101 \\ E. O. Lawrence Berkeley National Laboratory \\ Berkeley, CA 94720 \\ ooguri@physics. berkeley.edu
}

\begin{abstract}
We discuss issues concerning $\mathrm{M}$ (atrix) theory compactifications on curved spaces. We argue from the form of the graviton propagator on curved space that excited string states do not decouple from the annulus D0-brane $v^{4}$ amplitude, unlike the flat space case. This argument shows that a large class of quantum mechanical systems with a finite number of degrees of freedom cannot reproduce supergravity answers. We discuss the specific example of an ALE space and suggest sources of possible higher derivative terms that might help reproduce supergravity results.
\end{abstract}

February 1997 


\section{Introduction}

The conjectured description of $\mathrm{M}$ theory as matrix quantum mechanics [1] implies a substantial reduction in the apparent degrees of freedom necessary to describe $\mathrm{M}$ theory. A first reduction occurs because various different p-branes emerge as composites of of the $N$ D0-branes described by the $U(N)$ quantum mechanics as $N \rightarrow \infty$. The second reduction occurs because all excitations of the stretched string states connecting the D0-branes decouple from the leading low velocity dynamics, allowing classical supergravity interactions to emerge as the result of integrating out a finite number of quantum mechanical degrees of freedom. This phenomenon was found in the $v^{4}(v$ is the D0-brane velocity) leading weak coupling (annulus) amplitude in [2]. As explained very clearly in [3] it follows from the vanishing contribution of long (non-BPS) $N=4$ supersymmetry multiplets to this amplitude.

The scenario for proof outlined in [1] requires that i) all relevant velocities go to zero as $N \rightarrow \infty$ and ii) that there are no corrections beyond one loop in string theory to the leading low velocity dynamics. With these two conditions the decoupling of excited string states would extend to arbitrarily strong coupling. So the D0-brane $U(N)$ quantum mechanics which describes only the lowest unexcited stretched string state dynamics would provide an accurate strong coupling description. More generally, what is required is that the excited string states decouple from the full leading low velocity dynamics.

A priori, the excited state decoupling would be expected only for maximal (e.g. $N=$ $4, d=4)$ spacetime supersymmetry. More generally, the leading weak coupling $O\left(v^{4}\right)$ interaction crosses over from supergravity at distances $l>\sqrt{\alpha^{\prime}}$ to a sum over exchanges of all closed strings, equivalent to a quantum mechanical open string amplitude where all excited open strings contribute. At distances $l>>l_{P}^{11}$ (the eleven dimensional Planck length), this is essentially a one-loop amplitude.

In section 2, we show that this crossover is non-trivial in string theory whenever there is nonvanishing curvature in the compactified space. In other words, the form of the $O\left(v^{4}\right)$ interaction predicted by supergravity never agrees with the truncation of the one-loop open string amplitude result to a finite number of quantum mechanical degrees of freedom.

In section 3 we review the theory of D0-branes at weak string coupling on the orbifold $\mathbb{C}^{2} / \mathbb{Z}_{2}$ and its smooth resolution (Eguchi-Hanson space), developed in $|4|$. For present purposes, the main point is that this is a quantum mechanics with a finite number of degrees of freedom, to which the argument of section 2 applies. We compare the quantum 
mechanics and supergravity results and point out another mismatch with supergravity the mass of stretched open strings is apparently not proportional to their length - which may be resolved by further computation within the framework of [4].

In section 4, we outline a version of this definition which is well motivated at strong coupling (extending results of [5]), and we explain ways in which it might evade the theorem of section 2 .

We discuss some implications of these results in section 5 .

\section{Annulus}

We begin by computing the scattering of two $D 0$-branes on $R^{6} \times K 3$. We consider the case when they are fixed at points in $K 3$, but move on $R^{6}$ with relative velocity $v$ and impact parameter $b$. By combining results of [6], [7], [8] and [2], we find that their scattering amplitude is given by

$$
\mathcal{A}=\int \frac{d t}{t} e^{-t b^{2} / 2 \pi \alpha^{\prime}} \frac{1}{\eta(t)^{4}}\left(\frac{\theta_{11}^{\prime}(0 \mid t)}{\theta_{11}(\epsilon t \mid t)}\right) \times Z(t, \epsilon)
$$

where

$$
\begin{aligned}
Z(t, \epsilon)= & \operatorname{Tr}_{N S}\left(q^{L_{0}-1 / 4}\right) \frac{\theta_{00}(0 \mid t) \theta_{00}(\epsilon t \mid t)}{2 \eta(t)^{2}}-\operatorname{Tr}_{N S}\left((-1)^{F} q^{L_{0}-1 / 4}\right) \frac{\theta_{10}(0 \mid t) \theta_{10}(\epsilon t \mid t)}{2 \eta(t)^{2}}- \\
& -\operatorname{Tr}_{R}\left(q^{L_{0}}\right) \frac{\theta_{01}(0 \mid t) \theta_{01}(\epsilon t \mid t)}{2 \eta(t)^{2}}
\end{aligned}
$$

The parameter $\epsilon$ is related to the velocity $v$ as $\pi \epsilon=\operatorname{arctanh}(v)$. The traces

$$
\operatorname{Tr}_{N S}\left(q^{L_{0}-1 / 4}\right), \operatorname{Tr}_{N S}\left((-1)^{F} q^{L_{0}-1 / 4}\right), \operatorname{Tr}_{R}\left(q^{L_{0}}\right)
$$

are the partition function of the open string on $K 3$. The CFT of closed string on $K 3$ has two copies of the $N=4$ superconformal symmetry on the worldsheet. Since the D0-brane boundary condition preserves $1 / 2$ of them [9], at least one set of the $N=4$ superconformal algebra acts on the open string Hilbert spaces. We can therefore expand the open string partition functions in terms of the characters of the $N=4$ algebra with $\hat{c}=2$ studied in [10]. There are three types of representations of the $N=4$ algebra. Two of them have non-zero values of Witten index and are called the massless representations. Their 
conformal weights are 0 and $1 / 2$ and their characters $\chi_{0}$ and $\chi_{1 / 2}$ obey the following simple relation,

$$
\chi_{0}^{(N S)}+2 \chi_{1 / 2}^{(N S)}=\frac{q^{-1 / 8}}{\eta}\left(\frac{\theta_{00}}{\eta}\right)^{2}
$$

In particular, the Witten indices of the two representations cancel in this combination after performing the spectral flow to the R-sector. The third type is called a massive representation; it has no Witten index and it exists for any conformal weight $h>0$. The character of massive representation is

$$
\chi_{h}^{(N S)}=\frac{q^{h-1 / 8}}{\eta}\left(\frac{\theta_{00}}{\eta}\right)^{2}
$$

Now it is easy to see that the open string Hilbert space in question has no Witten index. This is because the string stretched between the two points on $K 3$ has a non-zero energy proportional to the geodesic distance between them. Although this is a semiclassical statement valid for distances much larger than the string length $l_{s} \sim \sqrt{\alpha^{\prime}}$, the fact that the Witten index vanishes is rigorous. Therefore the partition functions (2.3) of the open string should be given by

$$
\operatorname{Tr}_{N S}\left(q^{L_{0}-1 / 4}\right)=\frac{g(t) q^{-1 / 8}}{\eta}\left(\frac{\theta_{00}}{\eta}\right)^{2}
$$

where $g(t)$ encodes multiplicities of the $N=4$ algebra representations,

$$
g(t)=\sum_{i} q^{h_{i}}
$$

Substituting this into (2.2), we find

$$
Z(t, \epsilon)=\frac{g(t) q^{-1 / 8}}{2 \eta(t)^{5}}\left[\theta_{00}(0 \mid t)^{3} \theta_{00}(\epsilon t \mid t)-\theta_{10}(0 \mid t)^{3} \theta_{10}(\epsilon t \mid t)-\theta_{01}(0 \mid t)^{3} \theta_{01}(\epsilon t \mid t)\right] .
$$

When $v$ is small, we can expand (2.1) in powers of $\epsilon \sim v / \pi$. The $v^{2}$ term vanishes as in the case of the flat space [7] since

$$
Z(t, \epsilon)=O\left(v^{4}\right)
$$

Using the result of [2], the coefficient of the $v^{4}$-term can be expressed as

$$
\mathcal{A}_{v^{4}}=\int \frac{d t}{t} e^{-t b^{2} / 2 \pi \alpha^{\prime}} g(t) \prod_{n=1}^{\infty}\left(1-q^{n}\right)^{3}
$$


The question is whether only the lightest stretched string state contributes to $g(t)$. We now argue that this cannot be the case. To understand why, we observe that on general string theory grounds $\mathcal{A}_{v^{4}}$ approaches the result of massless closed string (supergraviton) exchange when the distances between the D0-branes are much larger than $l_{s}$. In this domain we have

$$
\mathcal{A}_{v^{4}} \rightarrow \int \frac{d t}{t}\left(\frac{t}{\alpha^{\prime}}\right)^{2} e^{-t b^{2} / 2 \pi \alpha^{\prime}}\left(e^{-\frac{\alpha^{\prime}}{t} \Delta_{K 3}}\right)_{x, y},
$$

where $\Delta_{K 3}$ is the Laplacian on $K 3$ and $x, y$ are points on $K 3$ where the D0-branes are located.

If (2.9) and (2.10) are to agree for all $b>>l_{s}$, we would require

$$
g(t)=\frac{\left(\alpha^{\prime} / t\right)^{2}}{\prod_{n=1}^{\infty}\left(1-q^{n}\right)^{3}}\left(e^{-\frac{\alpha^{\prime}}{t} \Delta_{K 3}}\right)_{x, y} .
$$

for all $t<<1$. This is not possible. To see this, let us expand the right-hand side for large $t$ (small $q=e^{-t}$ ). It can be done by using the adiabatic expansion of the heat kernel $e^{-\frac{\alpha^{\prime}}{t} \Delta}$, and we find

$$
g(t)=\frac{1}{\prod_{n=1}^{\infty}\left(1-q^{n}\right)^{3}} e^{-\frac{t}{\alpha^{\prime}} \sigma^{2}(x, y)}\left[1+\sum_{k=1}^{\infty} a_{k}(x, y)\left(\frac{\alpha^{\prime}}{t}\right)^{k}\right],
$$

where $\sigma(x, y)$ is the geodesic distance between $x$ and $y$, and $a_{k}(x, y)$ can be expressed in terms of the curvature of $K 3, \sigma(x, y)$, and their derivatives. We know that some of these coefficients are non-zero; in fact the first term is the Euler density. This expansion is valid for $t>>\sigma^{2} / l_{C}^{2}$ where $l_{C}$ is the characteristic curvature length. So for $l_{s}^{2}<<\sigma^{2}<<l_{C}^{2}$ there is a large region $1>>>>\sigma^{2} / l_{C}^{2}$ where (2.11) is valid. On the other hand, we know $g(t)$ must have an expansion of the form (2.7). No finite number of states, or discrete infinity of states whose gaps are not string scale or smaller, can reproduce the form (2.11) in the required range of $t$. On other hand the excited open string states can produce such effects and therefore must contribute. The unexcited multiple winding states important for describing D0-brane dynamics in toroidal compactifications [1, [1], or multiply wound extremal geodesic open strings, have gaps $\sim l_{C}^{2}$ and do not affect the above conclusions.

A similar argument to the above demonstrates that excited open string states must contribute in Calabi-Yau compactifications with spacetime $N=1$ supersymmetry as well. Again, the crucial point is that in general there is nonzero curvature in such compactifications, hence nontrivial power law corrections in (2.11) which cannot be reproduced by the unexcited open string states. 
Although the result is generic for the quantum mechanics of weakly coupled open strings, there are a number of implicit assumptions which might be violated in more general contexts, such as a quantum mechanics of $\mathrm{M}$ theory compactification. Perhaps the most serious is the decoupling between $R^{6}$ and $K 3$ world-sheet degrees of freedom. This led directly to the factorized nature of the amplitude (2.6), and the simple $b$ dependence in (2.9). In terms of quantum mechanics, it restricts the masses of states to depend on the parameters in $\mathbb{R}^{6}$ as

$$
m_{W}^{2} \sim b^{2}+f(x, y)
$$

with no explicit $v$ dependence. On the other hand, if we allow general dependence on $b$ and $v$, the supergravity result could be reproduced in many ways.

\section{Interactions of D0-branes on ALE space}

In [4] it was found that $N$ D0-branes on $\mathbb{C}^{2} / \mathbb{Z}_{2}$ are described by the dimensional reduction of $\mathcal{N}=1, d=6 S U(N) \times S U(N) \times U(1) \times U(1)$ gauge theory with two hypermultiplets in the $(N, \bar{N})_{(2,0)}$. The parameters $\zeta$ which blow this up to the Eguchi-Hanson space $\mathcal{M}_{\zeta}$ are simply the three Fayet-Iliopoulos terms for the non-trivial $U(1)$.

The strategy for defining D0-branes on an orbifold is identical in string theory and in $\mathrm{M}$ theory, but we briefly review it in the latter framework. We start with the maximally supersymmetric $U(2 N)$ quantum mechanics, and make a projection commuting with half of the supersymmetry,

$$
\begin{aligned}
\omega X & =\gamma X \gamma^{-1} \\
\omega^{-1} \bar{X} & =\gamma X \gamma^{-1} \\
A & =\gamma A \gamma^{-1}
\end{aligned}
$$

with $\omega=-1$ and $\gamma=\sigma_{3} \otimes \mathbf{1}_{N}$. Each boson has a partner fermion with the same projection. The bosonic matter is

$$
X=\left(\begin{array}{cc}
0 & b_{01} \\
b_{10} & 0
\end{array}\right) \quad \bar{X}=\left(\begin{array}{cc}
0 & \bar{b}_{10} \\
\bar{b}_{01} & 0
\end{array}\right) .
$$

The resulting Lagrangian is determined by the choice of gauge group and matter representation, if we assume the the matter Lagrangian is free before gauging. We will make this assumption, but discuss it further below.

We next review the identification of the Higgs branch of the moduli space with $\mathcal{M}_{\zeta}$. The analysis can be done for $N=1$; the complete Higgs branch for $N>1$ is the obvious 
symmetric product $\mathcal{M}_{\zeta}^{N} / S_{N}$. This is the hyperkähler quotient constructed by Kronheimer, defined by the three moment map (D-term) constraints

$$
\begin{array}{r}
b_{01} \bar{b}_{01}-b_{10} \bar{b}_{10}=\zeta_{C} \\
\left|b_{01}\right|^{2}-\left|b_{10}\right|^{2}-\left|\bar{b}_{01}\right|^{2}+\left|\bar{b}_{10}\right|^{2}=\zeta_{R}
\end{array}
$$

and the $U(1)$ gauge quotient. In the $\mathbb{Z}_{2}$ (Eguchi-Hanson) case, there is an $S U(2)$ symmetry under rotations of the vector $\vec{\zeta}$, allowing us to take $\zeta_{C}=0$ and $\zeta_{R}>0$ without loss of generality. We do so below.

The $\mathbb{P}^{1}$ produced by blowup is then

$$
b_{10}=\bar{b}_{01}=0
$$

Taking

$$
z_{1} \equiv b_{01} \quad z_{2} \equiv \bar{b}_{10}
$$

the remaining constraint and quotient become the usual Kähler quotient construction of the Fubini-Study metric on $\mathbb{P}^{1}$. Even more simply, we can generically gauge $\operatorname{Im} \bar{b}_{01}$ to zero, and the constraint becomes the usual $\sum x_{i}^{2}=\zeta$ defining $S^{2} \in \mathbb{R}^{3}$.

This quantum mechanics certainly falls under the hypotheses of the result in section two, and we conclude that the one-loop $O\left(v^{4}\right)$ interaction energy between the two D0branes cannot reproduce the subleading corrections in (2.11).

In fact the situation is worse - it does not reproduce the leading term. To see this, we compute the masses of the $\mathrm{W}$ bosons. On the Higgs branch, $U(2) \times U(2)$ is broken to $U(1) \times U(1)$, and thus these fall into 6 massive multiplets (hyper + vector) of $\mathcal{N}=2$. Each contains a massive vector boson whose mass matrix is $\operatorname{Tr}\left[\mathrm{A}^{\mathrm{i}},\langle\mathrm{X}\rangle\right]\left[\mathrm{A}^{\mathrm{j}},\langle\mathrm{X}\rangle\right]$, as in any Yang-Mills theory; all states in the multiplet have this mass.

This mass matrix is a truncation of that in the D-brane theory before applying the projection (3.1); furthermore gauge bosons with different eigenvalues under the projection operator $\gamma A \gamma^{-1}$ do not mix; therefore the mass of a stretched string is proportional to its length in the configuration space of the unprojected theory. Finally, since the vevs $\langle X\rangle$ are a linear subspace of those possible in the unprojected theory, this length is the same as the distance $\sqrt{\operatorname{tr}\left(X-X^{\prime}\right)^{2}}$ in the configuration space of the projected theory.

The conclusion is that the mass of a stretched string is proportional to its length in the larger configuration space. For the special case of two D0-branes located on the two-sphere (3.4), we can use symmetry to set $z_{2}=z_{2}^{\prime}=0$ leaving two positions $z_{1}$ and $z_{1}^{\prime}$ 
with $\left|z_{1}\right|^{2}=\left|z_{1}^{\prime}\right|^{2}=\zeta_{R}$. Then there are two massive multiplets with mass $\left|z_{1}-z_{1}^{\prime}\right|$ (the distance between the two branes), two with mass $2 \sqrt{\zeta_{R}}$ (the distance between a brane and its image), and two with mass $\left|z_{1}+z_{1}^{\prime}\right|$ (a brane and the other's image). In other words, the strings take the shortest path between D-branes (and their images), which passes inside the two-sphere, not on it. A shortest geodesic distance would of course be $\theta=\operatorname{Im} \log z_{1}^{\prime} / z_{1}$.

Thus we find the leading short distance behavior $\exp -\left|z_{1}-z_{1}^{\prime}\right|^{2} t / \alpha^{\prime}$ for the function $g(t)$ in (2.9), in contradiction with the leading term exp $-\sigma^{2} t$ in (2.11). Now this result is not in obvious contradiction with physical expectations for sub-stringy physics at weak string coupling. In principle, exchange of massive closed strings could combine to this answer. However, it is suspicious.

In fact, the result depends on an assumption which has not been proven: that the kinetic term in the world-volume gauge theory is the trivial $\sum\left|b_{i}\right|^{2}$. This is the simplest guess at a metric which on grounds of supersymmetry must be hyperkähler and admit a $S U(N) \times S U(N) \times U(1)$ isometry, but it has not been proven that it is the unique candidate. From world-sheet considerations along the lines of [4], the metric will be flat at $\zeta=0$ with corrections computable in weak string coupling. This computation and the question of whether it will modify this result are presently under study.

\section{D0-branes on ALE space at strong coupling}

In studying the same system at strong string coupling, we are forced to rely more on consistency arguments.

One natural idea is to start with gauge theory with a curved target space and dimensionally reduce it. This is interesting but will almost certainly produce a singular theory in the orbifold limit and as such is not likely to be the correct definition for small blow-up parameter.

Another natural approach which works well in the case of toroidal compactification is to introduce images under the space group. In the case of ALE this is exactly what we $\operatorname{did}$ above in defining $\mathbb{C}^{2} / \mathbb{Z}_{2}$. Clearly the correct supergravity interactions are obtained at one loop - they are the sum of image contributions.

The second step of adding the FI terms to produce the blowup (as proposed in [5]) is motivated by the observation that with this amount of supersymmetry there is nothing else we can do that changes the topology of moduli space. However, higher order corrections 
to the Lagrangian are less restricted. 1 In the orbifold limit, the lack of any scale (other than the overall coupling $\left.l_{P}^{11}\right)$ makes it very plausible that such corrections vanish.

To work around the better controlled orbifold limit, we can consider the blow-up to be accomplished by adding a condensate of particles in the multiplet associated with the

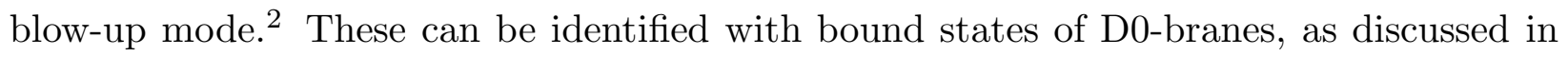
[5]. Although in the IMF it is not possible to make a spatially independent condensate this way, the minimal accessible longitudinal momentum $p_{-}$goes to zero (as $1 / N$ ) in the large $N$ limit of [1], and the result is effectively a constant blow-up for our purposes. For $\mathbb{C}^{2} / \mathbb{Z}_{2}$, the minimal bound state which respects the $U(1)$ manifest in the geometric description is a bound state in the $N=1$ model.

The condensate is most simply described by introducing second quantized operators which relate Hilbert spaces of different $N$. A creation operator $B^{+}$for the bound state we mentioned would act on the $N$ particle Hilbert space and produce a state in the $N+1$ particle Hilbert space. In the limit where the $N$ preexisting particles are far from the fixed point, the operation is simply tensor product. We will not attempt a general definition here (which probably requires knowing the bound state wave function) but make the assumption that correlations between different bound state (and other) particles can be neglected for our purposes. Then the wave function can be taken as a tensor product, and expectation values will add in a simple way. We then define an annihilation operator $B$ as its adjoint. The condensate is then

$$
|\zeta\rangle=e^{\zeta B^{+}}|0\rangle
$$

Given this definition, the bound state wave function, and the assumption that correlations can be neglected, it is straightforward in principle to deduce the Lagrangian describing D0-branes on the blown-up orbifold. The Fayet-Iliopoulos term must come from evaluating the potential $\sum_{i<j} \operatorname{tr}\left[X^{i}, X^{j}\right]^{2}$, with a vacuum expectation value for the off-diagonal components $\left[X^{i}, X^{j}\right]$. It would be interesting to verify this and it may be possible to find a topological quantity containing this expectation value by writing a trace projected to the BPS states, something like $\operatorname{Tr}(-1)^{\mathrm{F}} \mathrm{F}\left[\mathrm{X}^{\mathrm{i}}, \mathrm{X}^{\mathrm{j}}\right] \mathrm{J}_{\mathrm{R}}^{\mathrm{a}}$ where $J_{R}^{a}$ is the $S U(2)_{R}$ generator.

\footnotetext{
1 The explicit claims of [5] did not depend on these.

2 This idea and the related idea discussed in the conclusions arose in conversation with Tom
} Banks. 
This procedure can generate fairly general higher order corrections to the Lagrangian, with coefficients analytic in $\zeta$. As at weak string coupling, the first question of interest is whether corrections to the metric on moduli space can produce an effective Lagrangian for which stretched strings have mass proportional to geodesic length. In the present case, we have strong physical reasons to expect this, and the computation we have outlined will provide a significant test of this proposal.

Let us assume that this works, and ask whether the resulting theory can evade the result of section 2. Since the condensate is constant in the transverse dimensions, we expect a weaker version of the condition (2.12) to hold,

$$
m_{i}^{2} \sim b^{2}+f_{i}(x, y, v)
$$

However there is no reason for the theory to satisfy the stronger world-sheet decoupling condition described there.

One can imagine one loop diagrams which could reproduce higher order terms in the expansion (2.11). A term $a_{k} / t^{k}$ must contain $k$ fewer propagators for the states of mass squared $m^{2}=b^{2}+\sigma^{2}$, to make it less singular. Another necessary ingredient is that any singularities of the coefficients $a_{k}$ in the orbifold limit must come from integrating out states (since the orbifold limit is non-singular). This suggests that states of mass squared $m^{2}=b^{2}+\zeta$ must be present in the model, and indeed they are.

The tentative conclusion is that this model might reproduce the supergravity interaction, and work on testing this continues.

\section{Discussion}

In section 2 we have argued that no truncation to a finite number of open string degrees of freedom can reproduce graviton exchange in the annulus when the compactification has nonzero curvature. This means that the quantum mechanics obtained by truncating to the unexcited open string state will also fail to reproduce gravity at one loop. The "mild" infinity of wrapped open strings introduced in toroidal compactification [1, 11] also do not affect the result. In principle, they might bring in the need for a cutoff and renormalization, which could complicate the discussion. It is interesting that the explicit example in section 3, regarded as a 3-brane theory, is a finite quantum theory (as was noted in [4). By the general connection between open string UV and closed string IR limits, this might be 
expected as a general feature of theories with no closed string IR divergences, e.g. with more than four non-compact dimensions.

Higher order loop corrections in the string coupling $g_{s}$ are, by the scaling discussed in [12,13,2], an expansion in $\left(l_{P}^{11} / r\right)^{3}$ where $l_{P}^{11}$ is the eleven dimensional planck length and $r$ is a characteristic separation length $(\sim b, \sigma)$ of the D0-branes. As long as the curvature length $l_{C}$ is large compared to $l_{P}^{11}$ these effects will not affect the arguments in section 2 .

The argument clearly applies to the quantum mechanical model discussed in section 3 and in fact to a wide class of quantum mechanical models. The main assumptions are that there be a finite number of degrees of freedom, and restrictions on the higher derivative terms, particularly couplings to the velocity.

What kind of matrix model description might work for such compactifications? If the relevant velocities remain low one could recover the correct supergravity by adding explicit higher derivative terms to the quantum mechanics. A rather simplistic example would be to include the explicit $v^{4}$ term resulting from integrating out the excited open string states in the annulus. By itself this is probably not suitable (it would be singular in the orbifold limit), but some combination of explicit and induced interactions may well work, and the expansion in the blowup $\zeta$ described in section 4 might provide a theory of this type.

Large $N$ effects might provide another way for quantum mechanics to reproduce supergravity. If the matrix model description is correct in flat space it describes gravitons as bound states of D0-branes, so we expect a compactification could be represented as some kind of condensate of D0-branes. In section 4 we discussed such a condensate, and suggested that its effects could be summarized in an effective Lagrangian for a finite degree of freedom system. This is not logically necessary and it is also possible that the only probes which have correct $M$ theory physics are bound states of large numbers of D0-branes in the large $N$ limit. Perhaps the extended nature of the bound states enters crucially in this physics.

In any event it does seem that any matrix model description of $\mathrm{M}$ theory on curved spaces will be rather more intricate than the flat space description of [1].

\section{Acknowledgments}

We would like to thank Tom Banks, Greg Moore, Hiraku Nakajima, Nathan Seiberg, and Lenny Susskind for valuable discussions and correspondance. H.O. thanks the High Energy Theory Group of Rutgers University, where a part of this work was done, for hospitality. H.O. is supported in part by NSF grant PHY-951497 and DOE grant DEAC03-76SF00098. The work of M.R.D. and S.H.S. was supported in part by grant No. DE-FG02-96ER40959. 


\section{References}

[1] T. Banks, W. Fischler, S. Shenker, S. Susskind, "M Theory as a Matrix Model: a Conjecture," hep-th/9610043.

[2] M. R. Douglas, D. Kabat, P. Pouliot and S. H. Shenker, "D-Brane and Short Distances in String Theory," hep-th/9608024.

[3] C. Bachas and E. Kiritsis, "F(4) Terms in N=4 String Vacua", hep-th/9611205.

[4] M. Douglas and G. Moore, "D-Branes, Quivers, and ALE Instantons," hep-th/9603167.

[5] M. R. Douglas, "Enhanced Gauge Symmetry in M(atrix) Model," hep-th/9612126.

[6] J. Polchinski, "Dirichlet Branes and Ramond-Ramond Charges," Phys. Rev. Lett.75 (1995) 4724, hep-th/9510017.

[7] C. Bachas and M. Porrati, "Pair Creation of Open Strings in an Electric Field," Phys. Lett. 296B (1992) 77, hep-th/9209032; C. Bachas, "D-brane Dynamics," Phys. Lett. B374 (1996) 37, hep-th/9511043.

[8] M. R. Douglas and M. Li, "D-Brane Realization of $N=2$ Super Yang-Mills Theory in Four-Dimensions," hep-th/9604041.

[9] For CFT discussion of $D$-brane on $K 3$ or Calabi-Yau manifolds, see: H. Ooguri, Y. Oz and Z. Yin, "D-Branes on Calabi-Yau Spaces and Their Mirrors," Nucl. Phys. B477 (1996) 407, hep-th/9606112.

[10] T. Eguchi and A. Taormina, "Character Formulas for the $N=4$ Superconformal Algebra," Phys. Lett. 200B (1988) 314; "On the Unitary Representations of $N=2$ and $N=4$ Superconformal Algebras," Phys. Lett. 210B (1988) 125.

[11] W. Taylor, "D-brane Field Theory on Compact Spaces," hep-th/9611042; O. Ganor, S. Rangoolam and W. Taylor, "Branes, Fluxes and Duality in (M)atrix Theory," hepth/9611202.

[12] U. H. Danielsson, G. Ferretti, and B. Sundborg, "D-particle Dynamics and Bound States," hep-th/9603081.

[13] D. Kabat and P. Pouliot, "A Comment on Zero-Brane Quantum Mechanics," Phys. Rev. Lett. 77 (1996) 1004, hep-th/9603127. 